\title{
Féeries
}

Études sur le conte merveilleux, XVII $-\mathrm{XIXe}$ siècle

\section{Les Mille et Une Nuits en partage}

Sous la direction d'Aboubakr Chraïbi, Arles, Actes Sud, Sinbad, 2004, $524 \mathrm{p}$.

Jean Mainil

\section{(2) OpenEdition}

1 Journals

Édition électronique

URL : http://journals.openedition.org/feeries/130

DOI : $10.4000 /$ feeries. 130

ISSN : 1957-7753

Éditeur

UGA Éditions/Université Grenoble Alpes

Édition imprimée

Date de publication : 1 février 2005

Pagination : 290-296

ISBN : 2-84310062-3

ISSN : $1766-2842$

Référence électronique

Jean Mainil, «Les Mille et Une Nuits en partage », Féeries [En ligne], 2 | 2005, mis en ligne le 31 janvier 2007, consulté le 21 septembre 2020. URL : http://journals.openedition.org/feeries/130 ; DOI : https:// doi.org/10.4000/feeries. 130

Ce document a été généré automatiquement le 21 septembre 2020.

(c) Féeries 


\section{Les Mille et Une Nuits en partage}

Sous la direction d'Aboubakr Chraïbi, Arles, Actes Sud, Sinbad, 2004, $524 \mathrm{p}$.

\section{Jean Mainil}

1 Les Mille et Une Nuits en partage sont composées des actes du colloque « Mille et une nuits en partage » organisé par Aboubakr Chraïbi et qui s'est tenu à Paris en mai 2004 à la Fondation Singer-Polignac sous le patronage de l'Unesco, de la fondation SingerPolignac et du Cercle arabisant de recherche sur le monde arabe de l'InaLco. Ce colloque ainsi que le recueil critique auquel il a donné lieu célèbrent le tricentenaire de la parution, à partir de 1704, des Mille et Une Nuits d'Antoine Galland.

2 L'anthologie éditée sous la direction d'Aboubakr Chraïbi reprend en plus de cinq cent pages quelque trente-cinq articles par des spécialistes internationaux des Mille et Une Nuits. « Pour être mené à bien, le travail sur l'histoire des Mille et Une Nuits nécessite une approche interdisciplinaire ", suggère l'un des participants (D. Bodi, p. 395). C'est l'un des mérites de cette anthologie critique, et il n'est pas petit, d'avoir donné à des spécialistes de diverses origines, langues et surtout disciplines, l'occasion d'un dialogue sur l'incontestable chef-d'œuvre de littérature mondiale que constituent Les Mille et Une Nuits. C'est encore un autre mérite d'avoir permis un tel dialogue qui ouvre tant de nouvelles perspectives internationales et pluridisciplinaires sur Les Mille et Une Nuits tout en saluant la mémoire et l'œuvre de Galland.

L'Orientalisme, on le sait, n'a pas toujours, et n'a pas toujours eu, bonne presse. Sans remettre en cause les analyses spécifiques et pertinentes auxquelles la critique de l'orientalisme a conduit, l'approche qui est ici privilégiée souligne plutôt les spécificités textuelles et leurs évolutions à travers une approche pluridisciplinaire. Souvent "considérées comme une des principales pièces à conviction dans l'analyse de l'orientalisme", les traductions des Mille et Une Nuits sont bien souvent perçues « comme le texte orientaliste par excellence, collection de stéréotypes au service du marché culturel européen, qui s'intégra sans problème dans le corpus des visions européennes de l'Orient» (R. van Leeuwen, p. 121). Certes, personne ne niera que la création d'Antoine Galland s'écarte souvent des textes traduits en observant le code littéraire de l'époque où il écrit, ni qu'il ait aussi introduit «une vision de l'Orient 
absente des originaux " (S. Larzul, p. 260). Apparaissent ainsi de nombreux sofas qui démultiplient les références au suffa du texte original, mais dénotent aussi bien la mastaba (banc de pierre) dans des décors que complètent les inévitables eunuques et derviches (S. Larzul, p. 261). Aussi connaît-on le destin de ce Sopha, création au fond bien française et dont s'entichera le Siècle des Lumière à la suite de Crébillon qui emboîte ainsi le pas à Antoine Galland.

Jean-Paul Sermain commence son analyse des transitions dans Les Mille et Une Nuits par l'épisode suivant: grâce au génie de l'anneau, Aladdin sort du caveau où il était condamné à mourir de faim. Il est si faible qu'à peine arrivé chez sa mère, il s'évanouit de joie, mais aussi de faiblesse, cette «faiblesse dans laquelle il était de n'avoir pas mangé depuis près de trois jours ». La mère apporte tout ce qu'elle a, mais elle conseille à son fils d'user de patience: "Mon fils, lui dit-elle, ne vous pressez pas, cela est dangereux ; mangez peu à peu et à votre aise, et ménagez-vous dans le grand besoin que vous en avez [...] Aladdin suivit le conseil de sa mère ${ }^{1}$. « Sage lenteur ", commente J.-P. Sermain. Sage lenteur aussi, peut-être, que la célébration du tricentenaire de la traduction d'Antoine Galland qui a permis le recul nécessaire au double respect de l'original, ou plutôt des originaux, et du texte de Galland et de bien d'autres traductions et adaptations, comme le montrent avec autant d'érudition que de finesse les articles des Mille et Une Nuits en partage.

Délestées des approches parfois polémiques qui avaient tendance à cantonner l'œuvre d'Antoine Galland dans la catégorie d'orientaliste, mais sans pour autant nier que la construction d'un orient romanesque ait parfois aussi servi la construction d'une «conscience identitaire de l'Europe, en tant qu'il constitue la construction d'une antithèse » (R. van Leeuwen, p. 120), Les Mille et Une Nuits en partage pouvaient enfin, trois siècles après la parution de l'œuvre magistrale de Galland, montrer comment, synthèse de cultures anciennes de divers pays et de tant d'idéologies et de mentalités différentes, Les Mille et Une Nuits ont permis, depuis trois siècles, l'élaboration d'un «"autre" textuel » (R. van Leeuwen, p. 141), visuel et plus largement culturel.

6 S'il n'est plus nécessaire de prouver le caractère universel du chef-d'œuvre que constituent Les Mille et Une Nuits, il n'est cependant pas inutile - comme le font Les Mille et Une Nuits en partage - de rendre compte avec précision de l'immense richesse non seulement des sources qui ont influencé le chef-d'œuvre ou entretiennent avec elles des similitudes, tel «le plus ancien livre de l'humanité », L'Épopée de Gilgamesh (D. Bodi, p. 395), mais encore de montrer l'infini partage des Mille et Une Nuits. Des spécialistes rendent donc ici compte de ce fabuleux partage, l'immense influence des Mille et Une Nuits sur l'Europe (R. van Leeuwen et M. Sironval), sur la Grèce (H. Tonnet), sur l'Angleterre (Mushin al-Musawi) et sur le monde anglophone (The Arabian Nightmare de Robert Irwin analysé par Ferial Ghazoul). Mais le partage des Mille et Une Nuits ne s'arrête pas là : elles influenceront encore profondément le monde arabophone (M. Cassarino sur A. Kilito ainsi que "la mémoire collective arabe» telle qu'elle est analysée par Sobhi Boustani dans les poèmes de Khalîl Hâwî), en Italie, Pasolini et «le rôle de l'idéologie et de la subjectivité dans la constitution du texte cinématographique» (Wen-Chin Ouyang, p.85), au Japon (Tetsuo Nishio), et en particulier Mishima (Chikako Mori), et jusqu'à la poésie hébraïque amoureuse en Andalousie (M. Itzhaki).

$7 \quad$ Les Mille et Une Nuits, comme le rappelle Mohamed Agina, est un texte éminemment scriptible, pluriel, une «source d'écriture et de réécriture» (M. Agina, p. 268 et 274). 
Parmi ces réécritures, il est normal qu'une place importante revienne à celui qui a donné à la langue française ses Mille et Une Nuits puisqu'on fêtait en 2004 le tricentenaire de la parution du premier volume: Antoine Galland. En 1986, Georges May avait regretté que cette œuvre soit restée pour beaucoup un «chef-d'œuvre invisible » et avait essayé moins de réhabiliter le rôle du traducteur que de souligner ce qui, dans Les Mille et Une Nuits, procédait de l'adaptation, de la création. Il était donc question de "[revendiquer] pour Galland le titre d'écrivain à part entière, et, avec lui, la place qu'il nous semble mériter dans l'histoire de la littérature française ${ }^{2}$ ». Avec le précieux recueil d'Aboubakr Chraïbi et l'édition de Jean-Paul Sermain, la juste place d'Antoine Galland dans l'histoire de la littérature française lui a enfin été rendue pour dépasser le cadre même de cette littérature et rejoindre la littérature universelle.

Certes, comme le dit Aboubakr Chraïbi, "les littératures comme les sociétés sont représentatives de courants idéologiques multiples et contradictoires»(p.103). Il fallait donc que Galland, par la force des choses, de l'idéologie régnante, des goûts littéraires affichés à l'époque, adapte "au goût français » de la fin de l'époque louisquatorzienne les contes qu'il traduisait, quand il ne les développait pas à partir d'un scénario transcrit à partir d'un conte oral. Et c'est bien à ce génie de la création que de nombreux articles des Mille et Une Nuits en partage rendent hommage. A. Chraïbi montre par exemple comment l'idéologie informe la littérature à partir de l'épisode initial des Mille et Une Nuits : pour éviter que ne soit présente une adolescente (Dînârzâd) dans la pièce où un roi et son épouse sont en train de consommer leur mariage, Galland n'hésite pas à modifier la topographie de la chambre nuptiale de Shahriyâr. Selon Chraïbi, " il faudrait, idéalement, pour le destinataire, une deuxième femme qui fait semblant de la solliciter et de l'écouter" (p.102). Galland ne peut ni mettre l'adolescente en situation scabreuse, ni l'éliminer: non content de réaménager la chambre nuptiale, Galland utilisera encore une narrataire pour son propre ouvrage, poursuivant ainsi - idéologie oblige - le travail interne de son recueil et l'adressant à une autre femme. En miroir, suivant le manuscrit arabe qu'il utilise, mais tout en respectant la toute-puissante bienséance, Galland met en scène dans son recueil et son paratexte une «femme, sage et hautement cultivée, qui les raconte, et derrière une autre, d'un rang non moins élevé, qui les écoute » (p. 102).

La création de Galland, évidente quand il traduit, l'est bien entendu aussi, et a fortiori, quand il crée un conte à partir d'un autre conte qui lui a été fait et dont il a transcrit l'ébauche: il faut alors non seulement composer un conte à partir d'éléments disparates, mais il faut encore l'intégrer dans l'ensemble. Il ne suffit pas que se fasse une Nuit, encore faut-il qu'elle en devienne l'une des Mille et Une Nuits. Le génie de Galland consiste donc non seulement à rendre en français en le transformant un texte dont les références sont étrangères à la culture d'accueil mais aussi à respecter le " code littéraire en vigueur» (S. Larzul, p. 254) et l'esthétique classique: ce génie consistera donc encore à intégrer au récit-cadre chacune des Nuits. Dans cette optique, Jean-Paul Sermain analyse comment Galland développe un véritable art de la transition qui lui permet de répondre aux exigences des lecteurs de son époque en assurant la continuité narrative des Nuits, et de donner aux personnages une densité psychologique et sociale tout en écartant le danger d'une contamination par l'hétérogène et en évitant que, exigences génériques classiques obligent, le récit ne puisse être ramené au comique et à la dérision (p. 311). Comme point d'aboutissement, Jean-Paul Sermain analyse une des histoires dites « orphelines ", c'est-à-dire étrangère au recueil originel et qui, par les caprices d'un destin ironique, est devenue 
emblématique des Mille et Une Nuits : l'« Histoire d'Aladdin ». C'est, comme le montre Sermain, par sa manipulation des transitions que Galland peut mettre en scène un Aladdin qui « vit des moments si différents, qui est à la fois comique et sentimental, populaire et puissant, fourbe et galant» (p. 306). L'intégration de la figure d'Aladdin, absent du recueil originel, à un imaginaire universel montre à quel point Galland a réussi à donner à son personnage une "épaisseur sensible au lecteur" (306) tout en nous faisant voir les particularités les plus singulières de son personnage comme "l'expression particulière de ce qui est commun à chacun » (p. 312). Sermain montre donc comment, sous la plume de Galland, un personnage inconnu, orphelin en quelque sorte, entre au Panthéon des personnages mythiques universels.

L'immense succès que connut le chef-d'œuvre de Galland en France et à travers l'Europe eut moins pour effet, comme le suggère Richard van Leeuwen, de générer « un genre orientaliste, ou un ensemble cohérent d'idées sur l'Orient. Elle a en revanche stimulé le remodelage des genres européens [...] de nouvelles formes et de nouveaux concepts [...] Elle a trouvé sa voie dans le champ littéraire européen en refaçonnant les genres existants et en les transformant par sa capacité à bouleverser les conventions génériques et à forcer les auteurs à trouver de nouvelles formes" (p. 140). Inévitablement, la popularité et le caractère éminemment ouvert des Mille et Une Nuits ont conduit à la production d'un corpus orientalisant qui n'est sans soute pas étranger à la critique et à la qualification abusive du corpus en entier d'orientaliste : on produisit ainsi nombre de contes qui prenaient le contre-pied de Galland, malmenaient allègrement la pudeur, exaltaient la cruauté de fantasques sultans et la duplicité quasi nymphomaniaque de sultanes de pacotille. Loin de vouloir changer une loi inique qui condamne les femmes à mourir le lendemain de leurs noces pour un crime qu'elles n'ont pas commis, au lieu donc d'élever le conte au statut de contre-pouvoir qui finit par vaincre un pouvoir absolu, on en faisait désormais, à coup de Contes à dormir debout, le meilleur soporifique. Pour plaisants qu'ils soient (du moins certains), ces contes nous racontent évidemment moins l'Orient que les obsessions des contemporains des Lumières (et encore, du moins certains).

11 Mais Les Mille et Une Nuits ont aussi contribué, comme le suggère R. van Leeuwen, à un renouvellement en profondeur de formes narratives, comme le montre l'analyse de Jean-François Perrin. Signe de l'ouverture, de l'attrait et de la résonance des Mille et Une Nuits, c'est à un Écossais né en Irlande et francophone à qui reviendra le privilège d'utiliser les Mille et Une Nuits dans une production merveilleuse dont la poétique parodique et autoparodique est originale. Partant des contes de Galland et suivant la vague de l'orientalisme à la mode, Antoine Hamilton effectuera ainsi l'une des plus profondes remises en question des conventions narratives de son époque. Plus connu pour ses Mémoires du comte de Gramont, Hamilton est aussi l'auteur d'un corpus féerique oriental parodique et auto-parodique, principalement Le Bélier, Histoire de Fleur d'Épine et Les Quatre Facardins. Dans ces contes qui s'inspirent des Mille et Une Nuits, comme le montre Jean-François Perrin, Hamilton lance « des machines à déconstruire » à l'assaut des conventions littéraires de son époque, autant Modernes qu'Anciennes, pratiquant " une réception hypercritique » dans ses prologues versifiés, déconstruisant au passage le récit-cadre, passant d'un niveau à l'autre (traitement d'un motif épique en registre burlesque), implosant la narrativité même en superposant styles, modes et registres et en jouant «le fatras, l'entassement, l'accumulation, la digression, le coq-à-l'âne et l'absurde, contre les cohérences artificielles et les vraisemblances convenues » (p. 296). Jean-François Perrin analyse donc ici comment, avec l'œuvre parodique d'Antoine 
Hamilton, Les Mille et Une Nuits rejoignent cette autre épopée d'un autre voyageur dont l'attrait et la résonance sont aussi universels : Don Quichotte.

Il est impossible de rendre compte de toute la richesse de chacune des contributions. On mentionnera encore des approches plus formelles telles que les réflexions sur la constitution d'un index des passions et la tentative d'établir une grille de codage des Mille et Une Nuits « prises comme texte et non comme vestige documentaire renvoyant à des récits oraux antérieurs " par Claude Brémond (p. 30) ou encore la création d'une base de données des motifs des récits folkloriques par Jun'ichi Oda. D'autres articles montrent encore d'autres angles, qu'il s'agisse des textes antérieurs aux Mille et Une Nuits, de textes qui sont influencés par celles-ci, de films, de poésie, en Europe et dans de nombreux autres pays de diverses cultures et traditions. En plus de la qualité des contributions, ce que j'admire dans Les Mille et Une Nuits en partage et qui constitue à mon avis le plus grand hommage que l'on puisse faire à Galland, c'est que ce recueil a su, comme le savant arabisant, réunir en un tout cohérent et convaincant tant de voix, tant d'origines, tant de langues et d'approches théoriques diverses. À une époque où de nouveaux sultans rêvent d'imposer leur pouvoir, à notre époque où naissent de nouveaux orientalismes néo-colonisateurs sous l'égide d'un nouvel extrême-occident, il est devenu fondamental de se faire parler les cultures, d'écouter et de se parler, de se rappeler la puissance de la parole, et combien la tradition des Mille et Une Nuits est, et reste, profondément humaniste.

\section{NOTES}

1. Les Mille et Une Nuits, Contes arabes, traduction d'Antoine Galland, tome III, notice, chronologies, bibliographie et dossier par Jean-Paul Sermain, annexes par Aboubakr Chraïbi, Paris, GF Flammarion, 2004, p. 23.

2. Georges May, Les Mille et Une Nuits d'Antoine Galland, ou le chef-d'œuvre invisible, Paris, PUF, 1986, p. 67-68. 